\title{
Poly(2-isopropenyl-2-oxazoline) hydrogels for biomedical applications
}

Florica Adriana Jerca ${ }^{\mathrm{a}}$, Alina Maria Anghelache ${ }^{\mathrm{a}, \mathrm{b}}$, Emilian Ghibu ${ }^{\mathrm{a}}$, Sergiu Cecoltan ${ }^{\mathrm{c}, \mathrm{d}}$, Izabela Cristina $\mathrm{Stancu}^{\mathrm{b}, \mathrm{c}}$, Roxana Trusca ${ }^{\mathrm{e}}$, Eugeniu Vasile ${ }^{\mathrm{f}}$, Mircea Teodorescu ${ }^{\mathrm{b}}$, Dumitru Mircea Vuluga ${ }^{\mathrm{a}}$, Richard Hoogenboom ${ }^{\mathrm{g}}$ and Valentin Victor Jerca ${ }^{\mathrm{a}, \mathrm{g}^{*}}$

aCentre for Organic Chemistry “Costin D. Nenitescu”, Romanian Academy, 202B Spl. Independentei CP 35-108, 060023 Bucharest, Romania

${ }^{b}$ Department of Bioresorces and Polymer Science, Faculty of Applied Chemistry and Materials Science, University POLITEHNICA of Bucharest, 1-7 Gh. Polizu Street, 011061 Bucharest, Romania

${ }^{c}$ APMG - The Advanced Polymer Materials Group, Faculty of Applied Chemistry and Materials Science, University POLITEHNICA of Bucharest, 1-7 Gh. Polizu Street, 011061 Bucharest, Romania

Institute of Cellular Biology and Pathology "Nicolae Simionescu", Biopathology and Therapy of Inflammation, 8, B.P. Hasdeu Street, 050568 Bucharest, Romania

${ }^{\mathrm{e}}$ Faculty of Engineering in Foreign Languages, University POLITEHNICA of Bucharest, $313 \mathrm{Spl}$. Independentei 060042 Bucharest, Romania

fDepartment of Science and Engineering of Oxide Materials and Nanomaterials, University POLITEHNICA of Bucharest, 1-7 Gh. Polizu Street, 011061 Bucharest, Romania

${ }^{g}$ Supramolecular Chemistry Group, Centre of Macromolecular Chemistry, Department of Organic and Macromolecular Chemistry, Ghent University, Krijgslaan 281-S4, B-9000 Ghent, Belgium

ABSTRACT. Synthetic polymers have had a major impact on the biomedical field. However, all polymers have their advantages and disadvantages, so that the selection of a certain polymeric material always is a compromise with regard to many properties, such as synthetic accessibility, solubility, thermal properties, biocompatibility and degradability. The development of novel polymers with superior properties for medical applications is the focus of many research groups. The present study highlights the use of poly(2-isopropenyl-2-oxazoline) (PiPOx), as biocompatible functional polymer to develop synthetic hydrogel materials using a simple straightforward synthesis protocol. A library of hydrogels was obtained by chemical cross-linking of PiPOx, using eight different non-toxic and bio-based dicarboxylic acids. The equilibrium swelling degree (ESD) of the final material can be modulated by simple modification of the composition of the reaction mixture, including the polymer concentration in the feed ratio between the 2-oxazoline pendent groups and the carboxylic acid groups as well as the cross-linker 
length. The hydrogels with the highest water uptake were selected for further investigations regarding their potential use as biomaterials. We evaluated the thermoresponsiveness, the $\mathrm{pH}$-degradability under physiological conditions and demonstrated proof-of-concept drug delivery experiments. The in vitro cellular studies demonstrated the noncytotoxic character of the PiPOx hydrogels, and its protein repellent properties, while mineralization studies revealed that such scaffolds do not promote mineralization/calcification phenomena. In view, of these results, these hydrogels show potential use as ophthalmologic materials or in drug delivery applications.

\section{Keywords: poly(2-isopropenyl-2-oxazoline), hydrogels, pH degradability, thermoresponsive hydrogels}

\section{INTRODUCTION}

Over the past decades, polymer chemists have designed a wide range of synthetic biomaterials to be used in biomedical applications, and one particular class of biomaterials that has been the subject of intense research are hydrogels as they resemble natural materials, such as the extracellular matrix and the interior of cells. ${ }^{1-6}$ To date, extensive studies have been directed towards the development of synthetic hydrogels for wound dressing, ${ }^{7-9}$ tissue engineering, ${ }^{10-13}$ ophthalmology ${ }^{14,}{ }^{15}$ and drug delivery. ${ }^{16-20}$ Hydrogels are three-dimensional networks formed from hydrophilic homopolymers, copolymers, or macromers cross-linked to form water-insoluble polymer matrices. ${ }^{21}$ The hydrogels prepared via chemical cross-linking from synthetic hydrophilic monomers or polymers are often preferred over natural polymers due to their structural versatility, reproducibility of synthesis, controlled structure and improved mechanical properties. ${ }^{1,22-24}$ However the biocompatibility and biodegradability represent some major problems that still need to be tackled, especially in the case of implantable hydrogels. The majority of studies on synthetic polymer hydrogels are based on the use of poly(ethylene glycol)s (PEG), ${ }^{20,}{ }^{24}$ poly(vinylpyrrolidone) (PVP), ${ }^{25-27}$ poly(vinyl alcohol) (PVA), ${ }^{28-30}$ poly(hydroxyethyl methacrylate) (PHEMA), ${ }^{19,}$ 31-34 and more recently poly(2-oxazoline)s (PAOx). ${ }^{18,35-37}$ Although, these polymers have certain advantages they all come hand in hand with shortcomings that can limit their applicability as biomaterials. PEG has been the most commonly used water-soluble biomaterial for biomedical applications, because of the commercial availability in a wide range of molecular weights together with its hydrophilicity, solubility, biocompatibility, and reduced protein adsorption. ${ }^{38}$ However recent reports 
have shown that PEG can be nephro- and hepatotoxic, immunogenic with an incidence of allergy being present in a quarter of the population. ${ }^{39}$ Other concerns are the nonbiodegradable nature and the susceptibility to oxidative degradation that generates toxic products for the living tissues. ${ }^{38}$ PEG hydrogels are often designed from macromers with reactive end-chain functionalities, that allow limited covalent cross-linking loci. ${ }^{20}$ The chemical identity of the macromer and the mechanism of hydrogel formation are both important as each influences the cross-linking density of the hydrogel network and thus, dictates the value of the elastic modulus and the mesh structure. ${ }^{24}$ PVP is widely used in the development of hydrogel based-biomaterials, because it does not generally elicit an immune response, ${ }^{27}$ unlike poly(N-isopropyl acrylamide) (PNIPAM). ${ }^{40}$ However, potential disadvantages of PVP hydrogels include allergic reactions due to activation of suppressor $\mathrm{T}$ cells, ${ }^{41}$ nonbiodegradability, hygroscopicity ${ }^{42}$ and suppression of fertilization. ${ }^{43}$ Two other biocompatible polymers that are commonly used for developing hydrogels are PHEMA and PVA. Even though PVA is a biodegradable polymer with good mechanical properties in the dry state, its highly hydrophilic properties decrease the mechanical stability in the wet state. ${ }^{44}$ Moreover, many of the agents (i.e. glutaraldehyde, succinyl chloride) used for chemical cross-linking are cytotoxic, therefore limiting the biomedical applications or requiring laborious purification procedures. ${ }^{11,} 45$ PHEMA, has many advantages over other hydrogels such as: a water-content similar to living tissue, permeability to metabolites, resistance to absorption by the body and good optical transparency. ${ }^{1}$ However, PHEMA hydrogels are nondegradable in vivo, and their application in areas like tissue engineering has been restricted. Compared to the before mentioned polymers, the PAOx were more recently employed to develop hydrogel materials for biomedical applications. ${ }^{37,46}$ They can be regarded as interesting new hydrogels for biomedical applications, as PAOx exhibit good biocompatibility, hydrophilicity, and enable orthogonal functionalization possibilities, which has been well documented in recent years. ${ }^{18,35,37,47-52}$

Nevertheless, up to date there is no polymer that fulfills all the requirements for an ideal hydrogel with respect to its chemical nature, biocompatibility and biodegradability, and the chemical bond forming events to create covalent cross-links. Consequently, finding and investigating other alternative polymers represent the focus of the ongoing research.

2-Isopropenyl-2-oxazoline (iPOx) is a monomer belonging to the 2-oxazolines class, which via its 2-vinyl substituent can be polymerized to poly(2-isopropenyl-2-oxazoline) (PiPOx), ${ }^{53-55}$ with the retention of the 2-oxazoline ring as reactive side-chain functionality. Note that cationic ring-opening polymerization of iPOx is not possible due to interference of the isopropenyl group causing side reactions. ${ }^{56} \mathrm{PiPOx}$ is a 
versatile functional polymer as demonstrated in our recent reports ${ }^{53,57,58}$ due to the following reasons: i) PiPOx is highly hydrophilic, ii) is chemically inert to moisture and oxygen during storage, iii) is a functional polymer which can be prepared with well-defined characteristics by several living and controlled polymerization techniques, ${ }^{53,59-61}$ iv) the ring opening addition of the oxazoline side-chain functionalities requires simple post-modification reaction conditions, ${ }^{53,57,58} \mathrm{v}$ ) no catalysts are needed for the polymer analogous reaction, vi) no by-products are produced when reacted with carboxylic acids, ${ }^{53}$, 57, 58, 62 and vii) is biocompatible. ${ }^{62}$ Even though these characteristics make PiPOx highly appealing for the development of hydrogels for biomedical applications, to date there are no reports regarding PiPOx's potential to be used as basis for the development of chemically cross-linked hydrogels. However, to ideally meet the requirements for usage in biomedical applications, in general, hydrogels need to: i) possess readily tunable hydrophilicity, ii) have a highly reproducible composition via synthesis (functionalization or cross-linking degree), iii) exhibit biocompatibility and degradability, whereas the degradation products should be non-toxic; and iv) should have good mechanical properties in order to be processed and manipulated.

To tackle these aspects, we elaborated a two-step synthesis procedure to obtain hydrogels based on PiPOx. First we prepared a well-defined PiPOx with controlled molar mass and narrow molar mass distribution via anionic polymerization, following our latest optimized conditions ${ }^{53}$ (see Scheme 1). Then, we used the highly effective ring-opening addition reaction of the pendant iPOx rings with several nontoxic and bio-based dicarboxylic acids and prepared eight hydrogel series with different chemical compositions (see Scheme 1). Using this PiPOx post-polymerization modification approach, which was well documented in our group for soluble polymers, ${ }^{53,57,58,63}$ we ensure reproducible synthesis at any stage of the hydrogel preparation. Another advantage of this approach is that we can control the quantity of ester-amide cross-links generated during the post-modification reactions, in order to preserve the balance between hydrophilic character vs. structural integrity of the final hydrogels. Moreover, the esteramide cross-links generated by the ring opening addition, are susceptible towards degradation at basic and acidic $\mathrm{pH}$ values, and possibly to enzymatic degradation in in vivo environments. ${ }^{64}$

Thus, the present study aims to demonstrate that PiPOx represents a new versatile polymeric platform providing facile access to hydrogel materials with tunable water uptake. The hydrogels with highest water uptake are further investigated as thermoresponsive and side-chain degradable materials in simulated physiological buffer at different $\mathrm{pH}$ values. Preliminary investigations are carried out to 
evaluate the cytotoxicity, protein absorption and mineralization of these new materials. In addition, the potential of this hydrogels to be used in drug-delivery applications is addressed.

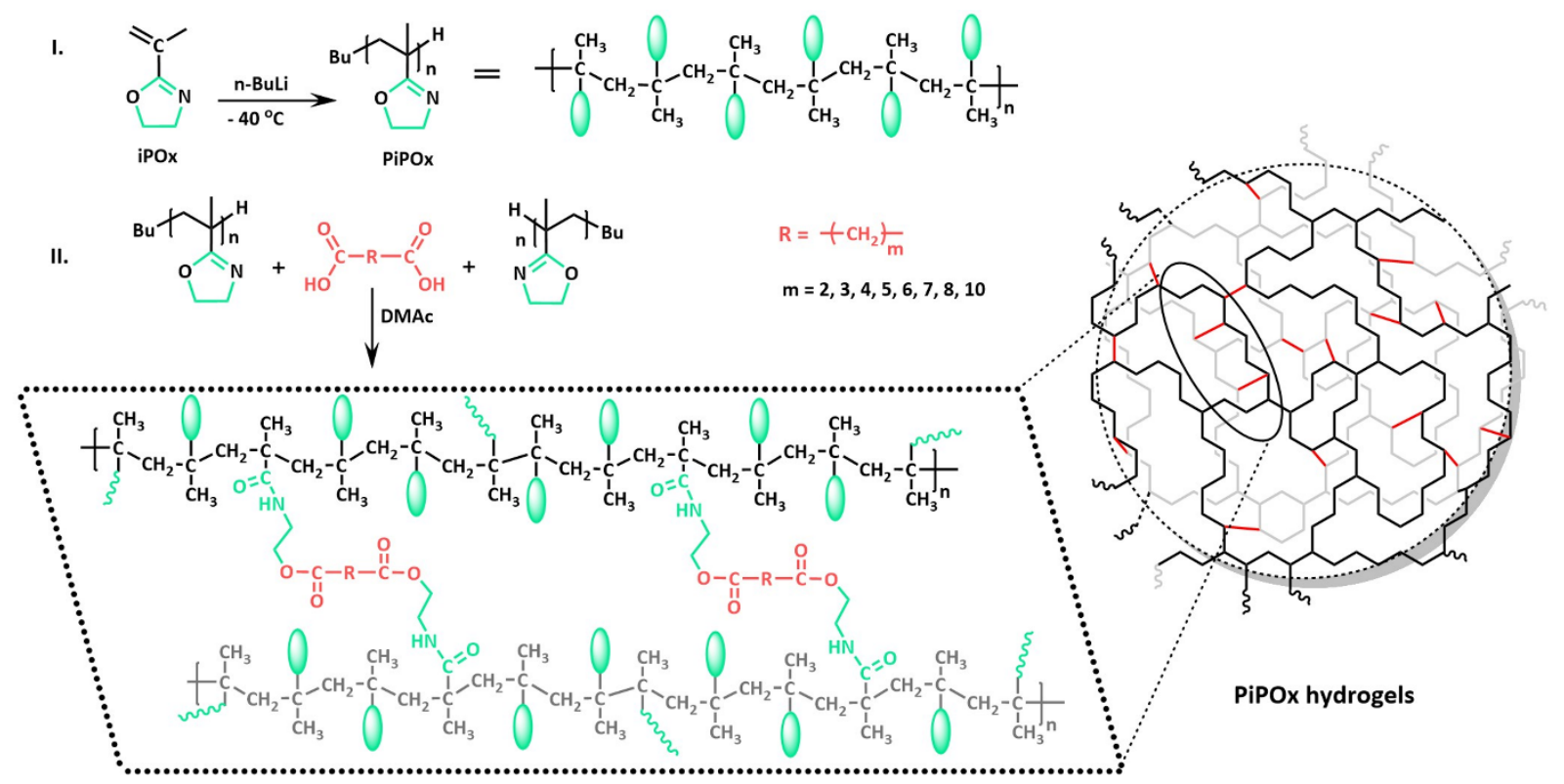

Scheme 1. Schematic representation of the reaction path to obtain PiPOx based hydrogels by cross-linking with dicarboxylic acids.

\section{RESULTS AND DISCUSSIONS}

\subsection{Synthesis and characterization of the PiPOx hydrogels}

To obtain cross-linked materials with tailored properties it is essential to control the chemical structure, dispersity and topology of the polymer. To ensure a high structural homogeneity of the hydrogels as well as reproducible properties and improved mechanical stability a well-defined polymer is needed. Therefore, making use of our recent optimized recipe for living anionic polymerization using commercially available n-BuLi, ${ }^{53}$ we synthesized a well-defined PiPOx homopolymer with a controlled number average molar mass of $10,600 \mathrm{~g} / \mathrm{mol}$ and a dispersity of 1.17 as determined by SEC with multi angle light scattering detector.

The cross-linked PiPOx networks were successfully obtained by reacting the side-chain 2oxazoline groups with 8 different dicarboxylic acids under catalyst free conditions in $N, N^{\prime}-$ dimethylacetamide (DMAc) at $140{ }^{\circ} \mathrm{C}$. The reaction temperature was chosen according to our previous 
studies regarding the ring opening addition reaction of PiPOx with different monocarboxylic acid to ensure a quantitative conversion of the 2-oxazoline rings and an optimum reaction time..$^{53,58}$

FT-IR analysis was used to investigate the chemical structure of the dry polymer networks. The FT-IR spectrum of PiPOx displayed the characteristic signals of the 2-oxazoline ring at $1648 \mathrm{~cm}^{-1}(\mathrm{C}=\mathrm{N}$ stretching) and $1121 \mathrm{~cm}^{-1}$ (C-O stretching) (see Fig S1). When the 2-oxazoline ring reacts with the acid, an ester-amide structure is generated (see Scheme 1). After cross-linking the signal corresponding to the carbonyl stretching vibration of the newly generated ester can be found at $1730 \mathrm{~cm}^{-1}\left(v_{\mathrm{C}=0}\right)$ (see Fig S1). In addition, although the carbonyl stretching vibration of the amide $\mathrm{I}$ band $\left(\mathrm{v}_{\mathrm{C}=\mathrm{O}}\right)$ at $1648 \mathrm{~cm}^{-1}$ overlaps with the $-\mathrm{C}=\mathrm{N}$ vibration of the 2-oxazoline ring, the amide II band appears as a distinct shoulder at 1530 $\mathrm{cm}^{-1}\left(v_{\mathrm{C}-\mathrm{N}}\right)$. The FT-IR data shows the successful modification reaction and revealed that all dry polymer networks have the same characteristic signals regardless of the cross-linker length (see Fig S1).

\subsection{Effect of reaction parameters on the hydrogel swelling ability}

The synthesis of hydrogels using the post-polymerization modification reaction of PiPOx emerges as an appealing chemical strategy due to several important advantages: i) biocompatibility of the starting polymer, ${ }^{62}$ ii) low toxicity of the bio-based dicarboxylic acids, especially the ones with longer alkyl chain (azelaic and dodecanedioic acid), iii) absence of catalyst and by-products, iv) reduce reaction time and v) the reaction is quantitative at $140{ }^{\circ} \mathrm{C}$. Finally, due to its versatility and robustness an entire library of hydrogels with tunable swelling behavior can effortlessly be obtained. The biomedical applications are predetermined by the capacity of hydrogels to absorb water. Therefore, since no literature studies could be found related to these materials we made a preliminary synthesis screening in which we investigated the influence of the following factors: (i) polymer concentration, (ii) molar ratio between the carboxylic acid ( $\mathrm{COOH}$ ) and 2-oxazoline (iPOx) groups; and (iii) chain length of the cross-linker on the equilibrium swelling degree (ESD) of the hydrogels. The swelling degree of the hydrogels in distilled water was measured at $20^{\circ} \mathrm{C}$ and calculated using equation $\mathrm{S} 1$, which is summarized in Table 1 .

The hydrogels were synthesized at four different polymer concentrations in DMAc: 10, 15, 20 and $25 \mathrm{wt} \%$. Equilibrium swelling degrees are obtained within $48 \mathrm{~h}$, and, as expected, are dependent on the polymer concentration in the feed. In the case of $10 \mathrm{wt} \%$ polymer concentration, bulk hydrogels could not be obtained, regardless of the acid that was used or the $\mathrm{COOH} / \mathrm{iPOx}$ molar ratio. However, we noticed an increase in the solution viscosity, probably due to mostly intramolecular reactions in combination with partial intermolecular coupling. At $15 \mathrm{wt} \% \mathrm{PiPOx}$ and a 0.075 molar ratio of $\mathrm{COOH} / \mathrm{iPOx}$ also no 
hydrogels could be obtained, whatever the acid used. However, the longer dicarboxylic acids yielded hydrogels at $15 \mathrm{wt} \%$ from 0.1 molar ratio of $\mathrm{COOH} / \mathrm{POx}$ while the shorter succinic acid did not result in hydrogels at $15 \mathrm{wt} \%$ at all. When using succinic acid as cross-linker, the first stable hydrogel (i.e. a hydrogel that can be cut into pieces, maintains its shape, is not sticky, and can be manipulated without breaking) was only obtained at $20 \mathrm{wt} \% \mathrm{PiPOx}$ and $0.1 \mathrm{~mole}$ ratio $\mathrm{COOH} / \mathrm{iPOx}$. The lower ability of succinic acid to form hydrogels with PiPOx may be ascribed to its shorter chain length, possibly favoring intramolecular coupling over intermolecular chain coupling. This hypothesis is confirmed by the fact that we initially also included the first two members of the dicarboxylic acid series (i.e. oxalic and malonic acid) in our studies. However, no shape stable hydrogels could be obtained even when the COOH/iPOx ratio was increased to 0.25 at $25 \mathrm{wt} \%$ polymer concentration. Therefore, in order to determine the structure of the formed material we removed the solvent, followed by washing the material with water and drying. FT-IR analysis revealed the same characteristic signals (see Fig. S2) as for the other hydrogels, which in combination with the inability to form hydrogels confirms that the coupling reaction mainly takes place intramolecularly due to the short chain length of the carboxylic acid.

From Table 1, one can notice that the ESD is higher at lower polymer concentration regardless of the cross-linker length, due to the formation of a more flexible network with lower cross-linking density. These results are in accordance with previous reports regarding the preferred formation of intramolecular loops at low reactant concentration. ${ }^{65}$ Furthermore, the ESD of the hydrogels decreased with increasing $\mathrm{COOH} / \mathrm{iPOx}$ molar ratio $(0.075,0.1$, and 0.125$)$, irrespective of the acid used as the cross-linker, (Table 1). This behavior can be explained by the fact that higher amounts of dicarboxylic acid lead to more crosslinked structures, which absorbs less water. Finally, the modification of the dicarboxylic acid chain length can have two opposite effects on ESD of hydrogels. Using an acid with a higher number of carbon atoms should increase the hydrophobic character of the hydrogel leading to a lower water uptake, while the crosslinking density should decrease generating a higher water absorption. The experimental results for the ESD demonstrated that the decrease in the cross-linking density predominates over the increase in the hydrophobic character especially in the case of longer chain acids (azelaic and dodecanedioic acid), whose water solubility is lower (Table 1). 
Table 1. The equilibrium swelling degree (ESD) of the hydrogel series, in distilled water at $20{ }^{\circ} \mathrm{C}$. Reported values are the mean of duplicate experiments provided with standard error.

\begin{tabular}{|c|c|c|c|c|c|c|c|c|c|c|c|}
\hline \multicolumn{2}{|c|}{$\begin{array}{l}\text { Acid } \\
\text { Name }\end{array}$} & \multirow{2}{*}{$\begin{array}{l}\mathbf{n}_{\mathrm{COOH}} \mathbf{n}_{\mathrm{iPOx}} \\
\text { Polymer } \\
\text { Conc. } \\
\text { (wt\%) }\end{array}$} & \multirow[b]{2}{*}{0.075} & \multirow[b]{2}{*}{0.1} & \multirow[b]{2}{*}{0.125} & \multicolumn{2}{|c|}{$\begin{array}{c}\text { Acid } \\
\text { Name }\end{array}$} & \multirow{2}{*}{$\begin{array}{l}\mathbf{n}_{\mathrm{COOH}}: \mathbf{n}_{\mathrm{iPO}} \\
\text { Polymer } \\
\text { Conc. } \\
\text { (wt\%) }\end{array}$} & \multirow[b]{2}{*}{0.075} & \multirow[b]{2}{*}{0.1} & \multirow[b]{2}{*}{0.125} \\
\hline 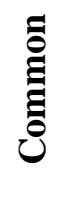 & $\stackrel{u}{ٍ}$ & & & & & 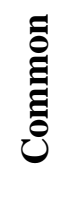 & $\underset{\Xi}{己}$ & & & & \\
\hline \multirow{4}{*}{ 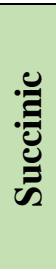 } & \multirow{4}{*}{ 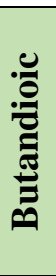 } & 10 & - & - & - & \multirow{4}{*}{ 元 } & \multirow{4}{*}{ 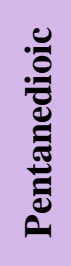 } & 10 & - & - & - \\
\hline & & 15 & - & - & - & & & 15 & - & $390 \pm 2$ & $319 \pm 2$ \\
\hline & & 20 & - & $\begin{array}{l}\mathrm{H} 4 \\
350 \pm 2 \\
\end{array}$ & $257 \pm 4$ & & & 20 & $\begin{array}{l}\text { H5 } \\
420 \pm 3\end{array}$ & $376 \pm 3$ & $312 \pm 4$ \\
\hline & & 25 & $364 \pm 3$ & $294 \pm 2$ & $238 \pm 3$ & & & 25 & $388 \pm 3$ & $343 \pm 2$ & $267 \pm 4$ \\
\hline \multirow{4}{*}{ 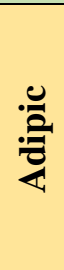 } & \multirow{4}{*}{ 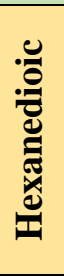 } & 10 & - & - & - & \multirow{4}{*}{ : } & \multirow{4}{*}{ 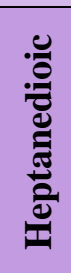 } & 10 & - & - & - \\
\hline & & 15 & - & $343 \pm 5$ & $311 \pm 4$ & & & 15 & - & $438 \pm 5$ & $344 \pm 3$ \\
\hline & & 20 & $\begin{array}{l}\text { H6 } \\
364 \pm 2\end{array}$ & $321 \pm 4$ & $291 \pm 3$ & & & 20 & $\begin{array}{l}\text { H7 } \\
506 \pm 2\end{array}$ & $416 \pm 4$ & $313 \pm 5$ \\
\hline & & 25 & $334 \pm 2$ & $245 \pm 3$ & $256 \pm 5$ & & & 25 & $444 \pm 3$ & $396 \pm 2$ & $283 \pm 4$ \\
\hline \multirow{4}{*}{ 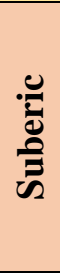 } & \multirow{4}{*}{ 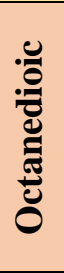 } & 10 & - & - & - & \multirow{4}{*}{$\frac{\stackrel{U}{\frac{U}{d}}}{\frac{\mathbb{N}}{4}}$} & \multirow{4}{*}{ 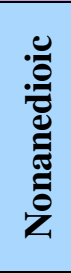 } & 10 & - & - & - \\
\hline & & 15 & - & $395 \pm 4$ & $316 \pm 2$ & & & 15 & - & $589 \pm 5$ & $467 \pm 3$ \\
\hline & & 20 & $\begin{array}{l}\text { H8 } \\
426 \pm 2\end{array}$ & $380 \pm 3$ & $300 \pm 2$ & & & 20 & $\begin{array}{l}\text { H9 } \\
675 \pm 1\end{array}$ & $522 \pm 5$ & $425 \pm 4$ \\
\hline & & 25 & $400 \pm 1$ & $365 \pm 4$ & $275 \pm 3$ & & & 25 & $600 \pm 5$ & $485 \pm 4$ & $315 \pm 3$ \\
\hline \multirow{4}{*}{ 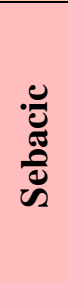 } & \multirow{4}{*}{ 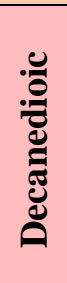 } & 10 & - & - & - & \multirow{4}{*}{ ' } & \multirow{4}{*}{ 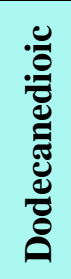 } & 10 & - & - & - \\
\hline & & 15 & - & $422 \pm 5$ & $345 \pm 2$ & & & 15 & - & $608 \pm 5$ & $470 \pm 4$ \\
\hline & & 20 & $\begin{array}{l}\text { H10 } \\
464 \pm 1\end{array}$ & $413 \pm 4$ & $331 \pm 4$ & & & 20 & $\begin{array}{l}\text { H12 } \\
732 \pm 3\end{array}$ & $555 \pm 4$ & $431 \pm 2$ \\
\hline & & 25 & $431 \pm 4$ & $377 \pm 3$ & $300 \pm 1$ & & & 25 & $620 \pm 2$ & $509 \pm 3$ & $320 \pm 5$ \\
\hline
\end{tabular}

In conclusion for this part, the hydrogel screening study proved that the ESD can be easily and accurately tuned by controlling the three parameters: polymer concentration, $\mathrm{COOH} / \mathrm{iPOx}$ molar ratio and chain length of the cross-linker, therefore paving the way for different applications from tissue engineering to ocular lenses that all require hydrogels with different water content.

Based on these data, a selection of the most promising materials for further investigations could be made. It is well known that hydrogels with higher water uptake are expected to release higher amounts of solutes and have a more pronounced $\mathrm{pH}$-degradability ${ }^{66}$ Therefore, the hydrogels with the highest ESD in their corresponding class, namely $\mathrm{H} 4, \mathrm{H} 5, \mathrm{H} 6, \mathrm{H} 7, \mathrm{H} 8, \mathrm{H} 9, \mathrm{H} 10$ and $\mathrm{H} 12$, were selected for further 
investigations. The conditions for these hydrogel preparations are $20 \mathrm{wt} \%$ polymer concentration and $0.075 \mathrm{~mol}$ ratio of $\mathrm{COOH} / \mathrm{PPOx}$, except for the succinic acid where the first stable hydrogel was obtained for $0.1 \mathrm{~mol}$ ratio of $\mathrm{COOH} / \mathrm{iPOx}$. Accordingly, we further investigated the network parameters, thermoresponsive behavior, and stability in simulated physiological fluids at different $\mathrm{pH}$ values of these hydrogels.

\subsection{Hydrogel network structure and odd-even effect}

In a next step, we examined the effect of the number of carbon atoms of the cross-linker on the network parameters, such as cross-linking density and mesh size as well as the ESD. From Table 2 it is evident that hydrogels cross-linked with dicarboxylic acids with an even number of carbon atoms have higher cross-linking density $\left(\rho_{c}\right)$ except for H12. The mesh size $(\xi)$ quantifies the distance between two adjacent cross-links and it is a critical parameter in controlling the drug diffusion rate as it reflects the amount of space available for a drug molecule to diffuse in or out of the swollen hydrogel network. Obviously, the mesh size follows the opposite trend of cross-linking density (i.e. the more cross-linked a hydrogel is the lower the mesh size).

Table 2. Network parameters for H4-H12 hydrogels.

\begin{tabular}{lllll}
\hline Code & $\begin{array}{l}\text { Number of } \\
\text { carbon atoms }\end{array}$ & $\bar{M}_{\boldsymbol{c}}(\mathbf{D a})^{\mathbf{a})}$ & $\boldsymbol{\rho c ~} \mathbf{~} \mathbf{1 0}^{-\mathbf{4}}\left(\mathbf{m o l} / \mathbf{c m}^{\mathbf{3}}\right)^{\mathbf{b})}$ & $\xi(\mathbf{n m})^{\mathbf{c})}$ \\
H4 & 4 & 755.9 & 14.03 & 13.33 \\
H5 & 5 & 988.2 & 10.74 & 18.42 \\
H6 & 6 & 801.3 & 13.24 & 14.29 \\
H7 & 7 & 1278.7 & 8.30 & 25.59 \\
H8 & 8 & 1008.4 & 10.52 & 18.89 \\
H9 & 9 & 1837.3 & 5.78 & 42.41 \\
H10 & 10 & 1138.6 & 9.32 & 22.02 \\
H12 & 12 & 2012.6 & 5.27 & 48.72 \\
\hline
\end{tabular}

a) average molecular weight between cross-links, calculated with equation S2

b) cross-linking density calculated with equation S4

${ }^{\text {c) }}$ mesh size calculated with equation S5

Consequently, the hydrogels cross-linked with dicarboxylic acids with even numbers of carbon atoms have lower ESD with respect to their odd analogs (Fig. 1). This effect can be probably assigned to a higher amount of cyclic defects present in the network of hydrogels crosslinked with odd dicarboxylic 
acids as previously reported by Olsen for polymer networks prepared via end-linking reactions. ${ }^{67}$ Using Monte Carlo simulation, they demonstrated an odd-even alternation of the loop densities on the junction functionality, which results from the topological constraint of the network. Even though the present hydrogels are not synthesized by end-linking coupling of polymeric precursor the same mechanism could operate also in our case. Consequently, the hydrogels cross-linked with dicarboxylic acids having odd numbers of carbon atoms have a lower crosslinking density due to the higher number of loops resulting in a higher ESD, as observed. Another noteworthy aspect is the notable increase of the ESD from 420\% (H5) to $675 \%$ (H9) registered in the case of odd alkyl ester amide hydrogels (see Table 1 and Fig. 1). While for the hydrogels with an even number of atoms the increase is shallow going from $350 \%$ (H4) to $464 \%$ (H10). However, the hydrogels cross-linked with dodecanedioic acid, having also an even number of carbon atoms, exhibited the highest ESD from the series. This occurrence can be explained by the lower crosslinking density of the hydrogel (i.e. higher $\xi$, see Table 2) due to increase flexibility of the crosslinker chain therefore producing a higher water absorption. Another aspect could be related to an increased probability to participate in intramolecular reactions because of the increased flexibility of the chain.

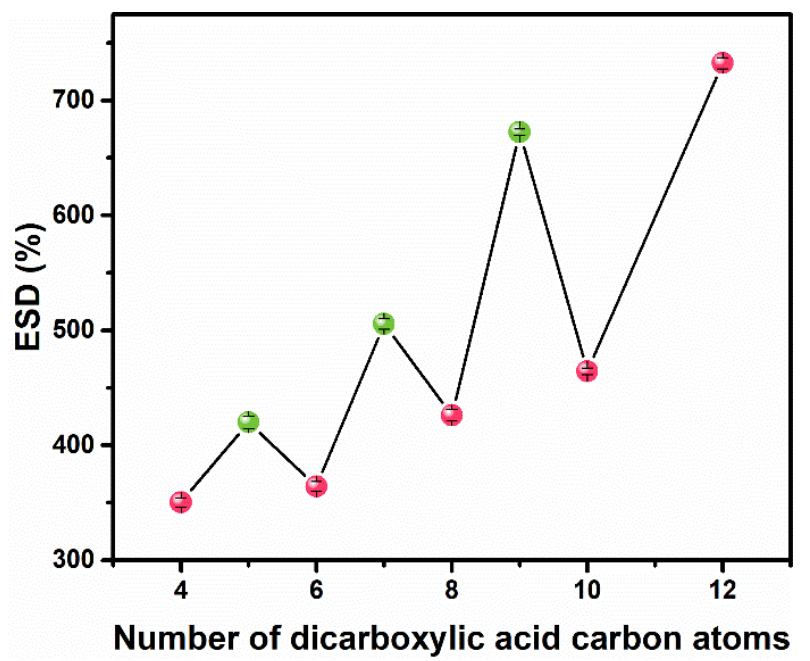

Figure 1. ESD dependence of the on the number of dicarboxylic carbon atoms in distilled water at $20^{\circ} \mathrm{C}$ for the hydrogels (H4-H12). Reported values are the average of duplicate experiments.

\subsection{Thermoresponsive properties of the H4-H12 hydrogels}

In a recent study, we demonstrated that PiPOx can be chemically modified with mono carboxylic acids yielding soluble thermoresponsive polymers. ${ }^{53,63}$ Modulation of the cloud point temperature was achieved by controlling the modification degree and/or by changing the hydrophobic character of the acid. 
Consequently, it may be expected that PiPOx based hydrogels containing longer hydrophobic ester amide chains to exhibit a volume-phase transition temperature (VPTT). The thermoresponsive behavior of the H4-H12 hydrogels was investigated by measuring their ESD at different temperatures. The temperature sweep experiments were carried out within the $20{ }^{\circ} \mathrm{C}-70{ }^{\circ} \mathrm{C}$ range. The ESD only slightly decreased in a continuous manner with increasing temperature for H4-H8 hydrogels indicating the absence of a VPTT (see Fig. 2). The slight decrease in water absorption with increasing temperature is a general phenomenon for hydrogels as the chain flexibility increases and the water-polymer interactions decrease. A more pronounced and abrupt decrease of ESD with temperature was noticed for $\mathrm{H} 9$ and $\mathrm{H} 10$ due to the increasing hydrophobic character of the dicarboxylic acid (see Fig. 2). However, the use of dodecanedioic acid led to a truly thermosensitive hydrogel with a VPTT of $43{ }^{\circ} \mathrm{C}$ (see Fig. 2). The larger transition interval may be ascribed to the random distribution of relatively large hydrophobic units ${ }^{68}$ by comparison with other thermosensitive hydrogels, such as poly $(\mathrm{N} \text {-isopropylacrylamide })^{69}$ or $\operatorname{poly}(\mathrm{N}$ vinylcaprolactam),${ }^{70}$ with smaller more evenly distributed hydrophobic domains that have a discontinuous volume phase transition, namely a narrow VPTT interval. However, increasing the content of acid, could lead to a VPTT close to body temperature and a narrow temperature transition with potential application in drug delivery, which is currently under investigation.
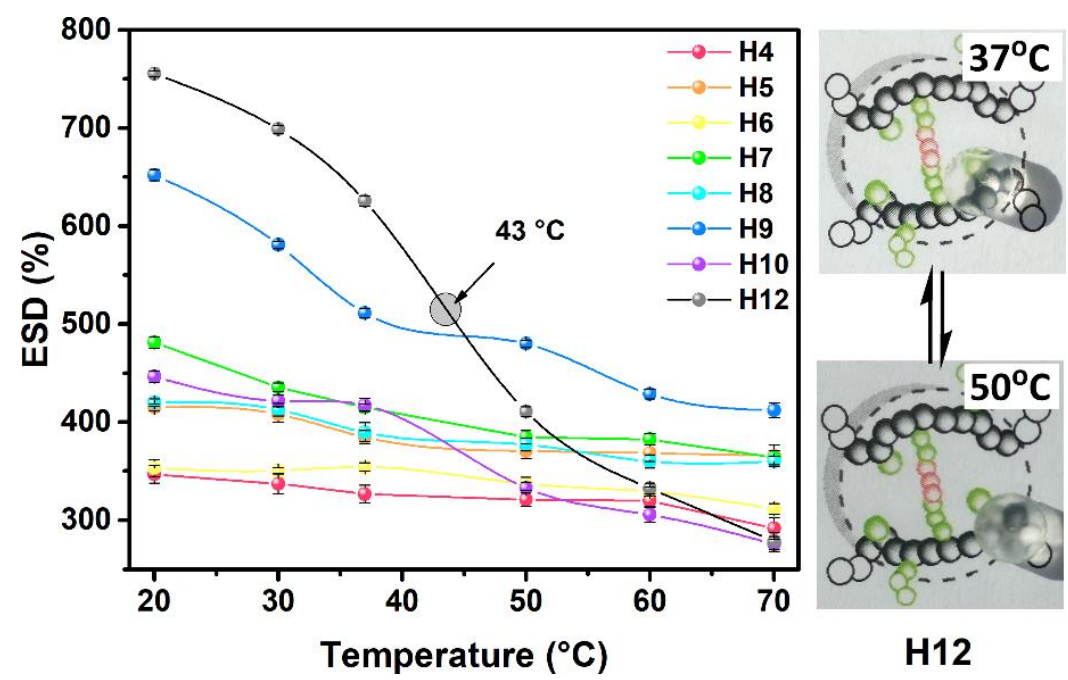

Figure 2. ESD dependence on temperature for the hydrogels (H4-H12) cross-linked with different dicarboxylic acids. Reported values are the average of duplicate experiments. The digital photos show the changes in transparency with temperature for the H12 hydrogel. 


\subsection{Stability in simulated physiological fluids}

To demonstrate the stability of the hydrogels at $\mathrm{pH} 7.4$, the samples were incubated in phosphate buffered saline (PBS) solution at $37^{\circ} \mathrm{C}$. The swelling ratios were calculated using equation (S1). The time needed for the hydrogels to reach the equilibrium swelling degree was similar for both water and PBS media, namely 48 hours (see Fig. 3) with a steady SD and dimensional stability over a 14-day period. Slightly lower ESD values were obtained in PBS compared to water, due to the presence of sodium chloride that has a salting out effect. However, the general odd-even trend is preserved in the H4-H12 series in PBS (see Fig S3).
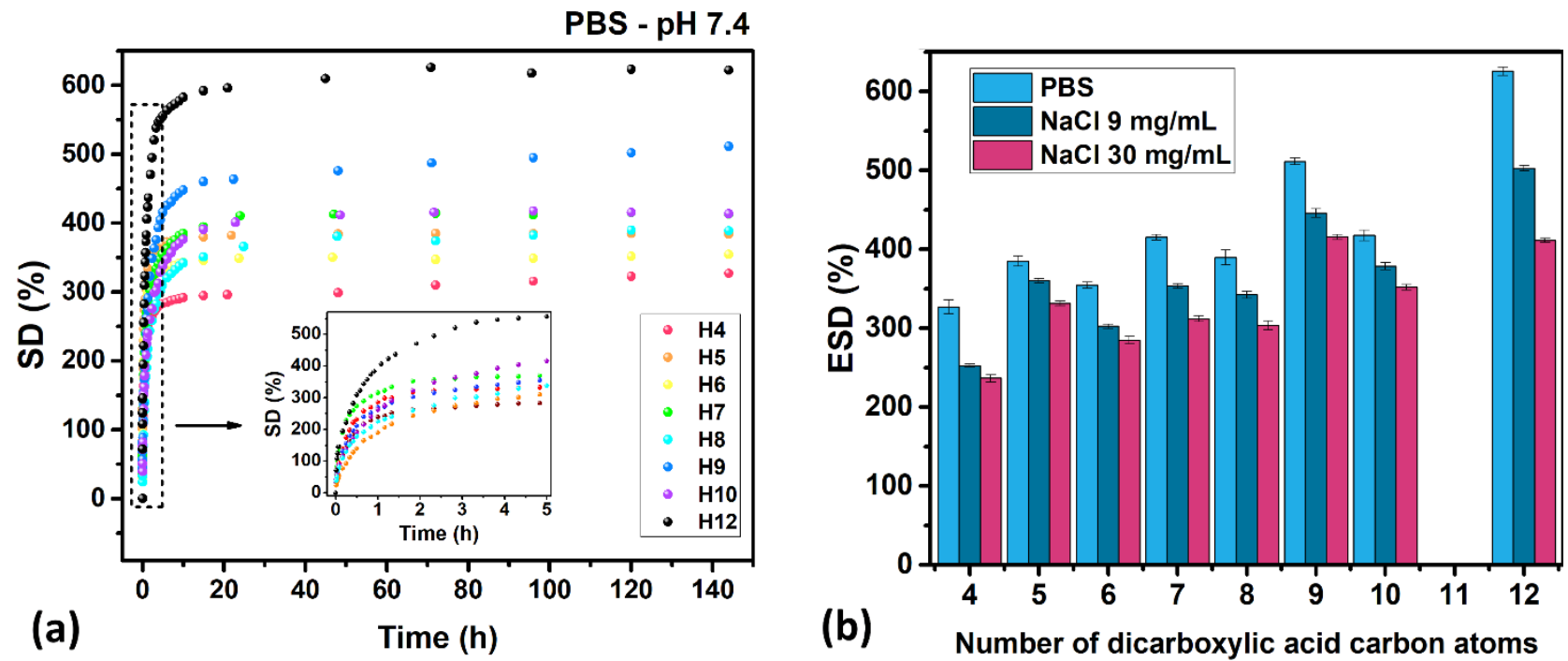

Figure 3. (a) Swelling degree versus time plots for the H4-H12 gels in PBS (pH 7.4) at $37^{\circ} \mathrm{C}$. Inset shows the swelling degree as a function of time for the first $5 \mathrm{~h}$ and (b) ESD dependence on saline solution concentration at $37{ }^{\circ} \mathrm{C}$ for the hydrogels (H4-H12). Reported values are the average of duplicate experiments.

In the view of biomedical applications, we tested also the swelling behavior of the H4-H12 gels in saline solutions. We choose two different concentrations of sodium chloride, namely $9 \mathrm{mg} / \mathrm{mL}$ and 30 $\mathrm{mg} / \mathrm{mL}$ corresponding to normal physiological saline solution and hypertonic saline, respectively. The results demonstrated that the presence of a salt in the swelling medium decreased the ESD of the H4-H12 hydrogels as compared to the results obtained in PBS (see Fig. 3b). The lower ESD can be ascribed to the salting out effect induced by the higher concentration of salting out ions compared to PBS. The ESD decrease was situated between 10\% and 14\% for $\mathrm{H} 5-\mathrm{H} 10$ gels, while the $\mathrm{H} 4$ and $\mathrm{H} 12$ hydrogels revealed 
a reduction of $22.4 \%$ and $19.5 \%$, respectively. Furthermore, the ESD was even lower for more concentrated salt solutions for all hydrogels (see Fig. 3a).

As previously stated, PiPOx hydrogels are designed to be susceptible to hydrolytic side-chain degradation through the ester bonds. The hydrolysis should lead to low-molecular weight products with low toxicity (i.e. dicarboxylic acids) and the generation of a soluble poly(2-isopropenyl-2-oxazoline-coN-(2-hydroxyethyl)methacrylamide) (co)polymer. The hydrolyzed moiety has a similar structure to poly(N-(2-hydroxypropyl) methacrylamide) (PHPMA), which is known to be biocompatible. ${ }^{71}$ Consequently, one may assume that if the molar mass of the starting PiPOx is lower than $\sim 40,000 \mathrm{~g} / \mathrm{mol}$ the resulting polymer could be excreted by renal filtration process. ${ }^{72}$ Therefore, the hydrolytic stability of the hydrogels was tested in two different buffer solutions with $\mathrm{pH}$ values of 3 and 11, respectively according to Mandal's protocol. ${ }^{73}$ Surprisingly, the water absorption of the hydrogels was lower at $\mathrm{pH} 3$ than at pH 7.4 irrespective of the cross-linker length (see Fig. 4a). For H4-H10 approximately a 1.3 fold decrease of the SD was registered in $24 \mathrm{~h}$. The highest difference in the SD of $~ 1.6$ fold lower was measured for H12. Moreover, the SD continuously decreased with time (see Fig 4a), although an opposite behavior was anticipated due to the cleavage of the ester bond. This anomalous behavior can be explained by the acid-catalyzed cross-linking of unreacted 2-oxazoline side chains, which lead to an increase in cross-linking density as illustrated in Scheme S1. The mechanism for this cross-linking is similar to that for the cationic ring opening polymerization of 2-oxazolines. ${ }^{48}$ Initial protonation of a pendant 2-oxazoline moiety generates an electrophilic site that may be attacked by the nucleophilic nitrogen of a neighboring 2-oxazoline ring, rapidly creating a ring-opened polymer network. To get more insight on the structure of the network formation on $\mathrm{pH} 3$ we removed the $\mathrm{H} 12$ hydrogel after 14 days of incubation washed it with distilled water and dried it. The FT-IR spectrum of the dry polymer network shows a decrease of the band at $1730 \mathrm{~cm}^{-1}$ compared to the gel incubated at $\mathrm{pH} 7.4$ proving that the cleavage of the ester takes place (Fig. S4). A closer inspection of the spectrum reveals a broadening of the band at $1650 \mathrm{~cm}^{-1}$ and the appearance of a new band at $1056 \mathrm{~cm}^{-1}$, corresponding to the newly formed cross-linked structure as proposed in Scheme S1. For comparison and clarity, we showed in Fig S4 also the spectra of the poly(2ethyl-2-oxazoline) obtained by cationic ring opening polymerization, which has a similar structure with the newly generated network. The ester hydrolysis was counterbalanced by the supplementary induced cross-linking of the iPOx unreactive groups, resulting in hydrogels which maintained their structural integrity (see Fig. S4 right photo). However, we cannot completely overrule the possibility of 2-oxazoline hydrolysis at this acidic $\mathrm{pH}$, which would generate a poly(methacrylic acid) copolymer. Ultimately this 
would also lead to a decrease in the ESD because the carboxyl groups are protonated at $\mathrm{pH} 3$. In view of these results, the side-chain degradability of soluble polymers will be a focus of our future work. This behavior can be further exploited for the synthesis of $\mathrm{pH}$ sensitive hydrogels.

At basic $\mathrm{pH}$ the SD continuously increased with time in the studied interval for all hydrogels. A higher water absorption, and in some cases, the complete dissolution of the hydrogel (H5-H9) into the incubation medium was registered due the ester bond cleavage leading to loss of the cross-links. Hydrogels that are cross-linked with acids that have an odd number of carbon atoms in the linker degraded much more rapidly and to a greater extent than the ones with even carbon atoms (see Fig 4b H7 and H9), except H5. One possible explanation for the faster degradation of $\mathrm{H} 7$ and $\mathrm{H} 9$ as compared to $\mathrm{H} 5$ could be their higher water absorption that increases the hydrolysis rate (hence the side-chain degradation). The increased stability of $\mathrm{H} 10$ and H12 hydrogels can be attributed to the hydrophobic shielding of the ester groups by the increase number of methylene units present in the crosslinker, thereby lowering the hydrolysis rate (Fig. S4 right photo). The SD of the $\mathrm{H} 4$ hydrogel increased with time while no fragmentation or dissolution was detected due to the higher cross-linking degree (see Table 1) as compared with the other hydrogels. Although at $\mathrm{pH} 11$ the hydrolysis of both amide and ester bond can occur, the FT-IR spectra proved that only cleavage of the ester is taking place. The intensity of the $1730 \mathrm{~cm}^{-1}$ band, corresponding to the $\mathrm{C}=\mathrm{O}$ ester vibration is reduced compared with the one in the starting dry network, while the amide II band $\left(1530 \mathrm{~cm}^{-1}\right)$ increases slightly and shifts to higher wavenumbers, probably due to the presence of salts from PBS (see Fig S4).
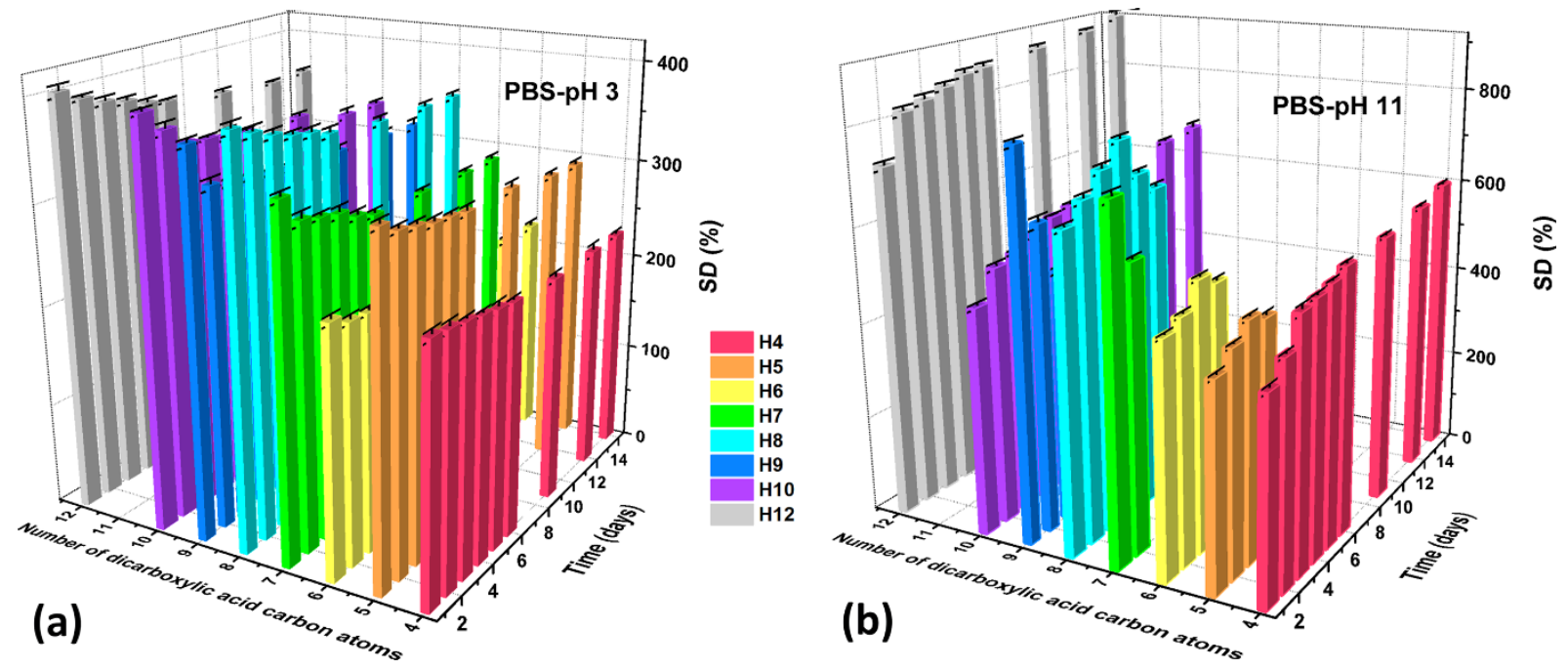

Figure 4. Swelling degree-time plots for the H4-H12 gels in PBS at $37{ }^{\circ} \mathrm{C}$ : (a) $\mathrm{pH} 3$ and (b) $\mathrm{pH} 11$. Reported values are the average of duplicate experiments. 


\subsection{Analysis of the swelling process}

For drug delivery applications, not only the swelling degree is important, but also the swelling rate as this may influence how fast a drug can be absorbed or released. Therefore, we investigated the mechanism of water transport and the swelling kinetics within the H4-H12 gels in PBS at $37{ }^{\circ} \mathrm{C}$. The swelling process of hydrogels can be divided into two molecular processes: penetration of the solvent molecules into the void spaces in the network and subsequent relaxation of the polymeric chains. The process can be analyzed by the Fickian diffusion model and the Schott's second-order-kinetic model, respectively. To determine the mechanism of water diffusion through the hydrogel in the initial swelling state, equation 1 was employed, considering only the data fulfilling the condition $\mathrm{SD}_{\mathrm{t}} / \mathrm{ESD} \leq 0.6{ }^{74}$

$\frac{M_{t}}{M_{\infty}}=\frac{S D_{t}}{E S D}=k \cdot t^{n}$

Where $\mathrm{M}_{t}$ and $\mathrm{M}_{\infty}$ represents the amount of water absorbed at time $\mathrm{t}(\mathrm{min})$ and at equilibrium, respectively, $\mathrm{k}$ is a constant depending on the characteristics of both gel and solvent, while $\mathrm{n}$ is the swelling exponent, indicating the diffusion mechanism. The constants $\mathrm{k}$ and $\mathrm{n}$ were determined from the intercept $(\operatorname{logk})$ and slope $(n)$ of the $\log \left(\mathrm{SD}_{\mathrm{t}} / \mathrm{ESD}\right)$ vs. $\log (\mathrm{t})$ plots (Fig. S5a). Depending on the relative rates of diffusion and polymer relaxation, three mechanism of diffusion can be distinguished for disk shape samples: i) Fickian diffusion mechanism (Case I) when $\mathrm{n}=0.5$, ii) non-Fickian (anomalous) diffusion mechanism for $0.5<\mathrm{n}$ $>1$ and finally iii) Case II diffusion mechanism when $\mathrm{n}=1$.

The calculated values for $\mathrm{k}$ and $\mathrm{n}$ are given in Table 3. For H4 - H8 hydrogels the swelling exponent was larger than 0.5 supporting the anomalous transport mechanism, i.e. the water uptake is controlled collaboratively by water diffusion and relaxation of the polymeric network. While the swelling mechanism of the H9-H12 hydrogels changes to diffusion controlled meaning that the water diffusion rate is much lower than polymer relaxation. This further suggests that the hydrogels cross-linked with longer alkyl chain dicarboxylic acids have a more flexible structure because of the lower cross-linking density. 
Table 3. Swelling kinetic parameters for $\mathrm{H} 4-\mathrm{H} 12$ hydrogels in PBS at $37^{\circ} \mathrm{C}$.

\begin{tabular}{llllllllll}
\hline & \multicolumn{4}{c}{ Fickian diffusion model } & \multicolumn{3}{c}{ Schott's second order kinetic model } \\
\hline Code & $\mathrm{n}$ & $\mathrm{k} \cdot 10^{2}$ & $\mathrm{R}^{2}$ & Diffusion mechanism & $\begin{array}{l}\mathrm{ESD}_{\exp } \\
(\mathrm{g} / \mathrm{g})\end{array}$ & $\begin{array}{l}\mathrm{ESD}_{\text {calc }} \\
(\mathrm{g} / \mathrm{g})\end{array}$ & $\begin{array}{l}\mathrm{K} \cdot 10^{3} \\
\mathrm{~g} /(\mathrm{g} \cdot \mathrm{min})\end{array}$ & $\mathrm{R}^{2}$ \\
\hline H4 & 0.529 & 10.35 & 0.9935 & Anomalous & 3.18 & 3.12 & 12.19 & 0.9989 \\
\hline H5 & 0.510 & 15.49 & 0.9921 & Anomalous & 3.85 & 3.86 & 23.43 & 0.9999 \\
$\mathbf{H 6}$ & 0.616 & 9.42 & 0.9943 & Anomalous & 3.54 & 3.49 & 22.82 & 0.9990 \\
H7 & 0.533 & 8.62 & 0.9953 & Anomalous & 4.15 & 4.13 & 7.26 & 0.9999 \\
H8 & 0.518 & 5.98 & 0.9986 & Anomalous & 3.90 & 3.83 & 4.84 & 0.9997 \\
H9 & 0.437 & 8.39 & 0.9989 & Fickian & 5.02 & 4.94 & 4.18 & 0.9979 \\
H10 & 0.416 & 9.90 & 0.9936 & Fickian & 4.17 & 4.20 & 4.74 & 0.9991 \\
H12 & 0.402 & 12.41 & 0.9952 & Fickian & 6.26 & 6.22 & 5.30 & 0.9994
\end{tabular}

To have an overall assessment of the diffusion mechanism during the entire swelling period, the Schott's second-order kinetic model must be used and it can be described using equation $2 .^{75}$

$$
\frac{t}{S D_{t}}=\frac{1}{K \cdot E S D^{2}}+\frac{t}{E S D}
$$

Where $\mathrm{SD}_{\mathrm{t}}(\mathrm{g} / \mathrm{g})$ and $\mathrm{ESD}(\mathrm{g} / \mathrm{g})$ are the swelling degree at time $\mathrm{t}(\mathrm{min})$ and the equilibrium swelling degree, respectively; $\mathrm{K}(\mathrm{g} / \mathrm{g} \cdot \mathrm{min})$ is the swelling rate constant.

The plots of $\mathrm{t} / \mathrm{SD}_{\mathrm{t}}$ versus time revealed perfect straight lines (Fig. S5b) with very good linear correlation coefficient $\left(\mathrm{R}^{2}>0.99\right.$, Table 3$)$ for all hydrogels, thus confirming that the Schott's secondorder-kinetic model can be effectively applied for describing the entire swelling process. The equilibrium swelling degrees $\left(\mathrm{ESD}_{\text {calc }}\right)$ determined from the plots, agreed very well with less than $2 \%$ error with the experimentally measured $\left(\mathrm{ESD}_{\exp }\right.$ ) (see Table 3). Analyzing the data in Table 3 we can observe that the K constant is larger for the hydrogels cross-linked with shorter carboxylic acids (H4-H6) that have higher hydrophilicity. The lower value found in the case of $\mathrm{H} 4$ can be explained by the higher degree of crosslinking (10 mol\% COOH/iPOx) which slows down the water diffusion process. For hydrogels cross-linked with longer alkyl chains dicarboxylic acids, the $\mathrm{K}$ constant is approximately 4 times lower and starts to level off, thus accounting for the increased hydrophobic character of the cross-linker.

\subsection{In vitro release of drugs}

The potential of the PiPOx hydrogels for drug delivery was preliminarily assessed using two different hydrophilic model drugs: 5-fluorouracyl (5-FU) and sodium diclofenac (DCFNa). DCFNa is a larger molecule than 5-FU and is also able to form hydrophobic interactions as well as hydrogen bonds, while 5-FU can form stronger hydrogen bonding but the capacity to form hydrophobic interactions is 
reduced. Moreover, two different hydrogels were chosen for these tests, H4 and H9, which we expected to interact in a different manner with the drugs resulting from the large difference in dicarboxylic acid chain length. The amount of drug released was determined by UV-Vis spectroscopy and calculated from the corresponding calibration curve prepared using standard solutions of known concentrations according to literature. ${ }^{76}$

The drug release experiments revealed a much higher release rate in the case of 5FU (Fig. 5), despite that the hydrogels had different mesh sizes $(\mathrm{H} 4, \xi=13.3 \mathrm{~nm}$ and $\mathrm{H} 9, \xi=42.4 \mathrm{~nm})$. One can conclude that the polymeric network interacts stronger with the DCFNa and a prolonged release is obtained. This behavior could be ascribed to the lower molecular size of 5FU and the reduced ability to form hydrophobic interactions. However, regardless of the drug used, the hydrogel H9 with a higher mesh size revealed slower drug release indicating that the hydrophobic interactions between the drug and the polymer network are more important for the release rate of small molecule drugs than the mesh size. Finally, the data suggests that a variable drug release rate can simply be obtained through variation of the cross-linker chain length.
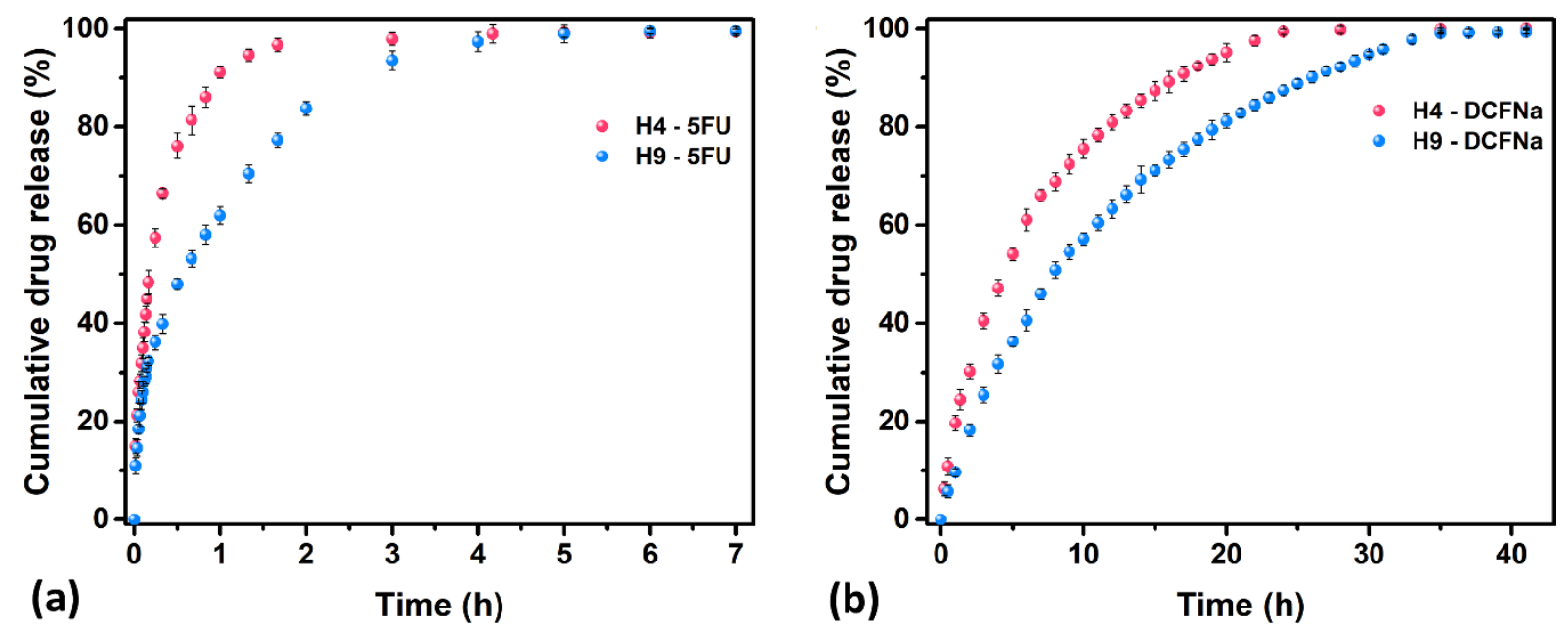

Figure 5. Influence of the dicarboxylic acid chain length on the in vitro release of: (a) 5-fluorouracyl $(5 \mathrm{FU})$ and (b) diclofenac sodium (DCFNa) in time. Data shown are averages of three release experiments.

Based on the cumulative release vs. time plots, the mass transport mechanism of the drug from the H4 and H9 hydrogels was analyzed by means of the Ritger-Peppas equation ${ }^{77}$ by considering only the data fulfilling the condition $\mathrm{M}_{\mathrm{t}} / \mathrm{M}_{\infty}<0.6$.

$\frac{M_{t}}{M_{\infty}}=k \cdot t^{n}$ 
Where $M_{t} / M_{\infty}$ is the fractional drug release after time $t(\mathrm{~min})$, while $\mathrm{k}$ and $\mathrm{n}$ are two constants, the exponent $\mathrm{n}$ being characteristic to the transport mechanism.

A linear correlation was found by plotting $\log \left(\mathrm{M}_{t} / \mathrm{M}_{\infty}\right)$ vs. $\log (\mathrm{t})$ and the slope of the lines was used to calculate the $n$ value (Fig. S6). The $n$ values for 5FU were lower than 0.5 thus indicating that the drug transport mechanism is influenced only by diffusion (Fig S6). On the contrary, for DCFNa an anomalous (non-Fickian) transport mechanism $(0.5<\mathrm{n}<1)$ was valid for both hydrogels (Fig S6). In this case the rates of drug diffusion and polymer relaxation were similar, and therefore both diffusion and gel swelling influenced the drug release.

\subsection{Direct cytotoxicity assay}

An important argument that moves PiPOx polymer forward to a variety of biological applications, is the recent report by Kronek's group concerning PiPOx's biocompatibility. ${ }^{62}$ Consequently, we investigated the cytotoxic nature of PiPOx using MTT cytotoxicity assay to determine if there is any difference between the polymer obtained by radical (Kronek's study) and anionic polymerization (present study). The cytotoxicity studies revealed that the non-toxic concentration for a cell viability higher than $80 \%$ is $13.5 \mathrm{mg} / \mathrm{mL}$ after $24 \mathrm{~h}$ of incubation (Fig. S7a). The results obtained are similar with those reported by Kronek. ${ }^{62}$ Furthermore, we performed a cytotoxicity assay of the hydrogels to assess their use as potential biomaterials. For this test we used the hydrogel cross-linked with azelaic acid. Culturing the L929 murine fibroblast cells on the hydrogel for 1-day led to a similar viability when compared to tissue culture plates surface, as seen in Figure S7b. No significant difference was found ( $p>0.6)$ confirming the high potential of this hydrogel for further investigations as biomaterial.

\subsection{Protein adsorption}

Next, we investigated the preliminary protein resistivity of the $\mathrm{H} 9$ hydrogel, at one model protein, BSA, since azelaic acid is a naturally occurring dicarboxylic acid that has antimicrobial activity and decreases hyperkeratosis, and has also been used for topical applications. ${ }^{78}$ The ability to repel proteins within aqueous environments would be extremely beneficial preventing many undesired polymer-cell and polymer-microorganism interactions, known as anti-fouling behavior. The amount of protein adsorption from $0.5 \mathrm{mg} / \mathrm{ml} \mathrm{BSA}$ and $1 \mathrm{mg} / \mathrm{ml} \mathrm{BSA}$ solutions was very small, close to the detection limit for the Coomassie colloidal blue G250 staining. The desorbed protein from $3 \mathrm{mg} / \mathrm{ml}$ BSA was estimated at $2.3 \pm$ $0.2 \mu \mathrm{g} / \mathrm{cm}^{2}$ hydrogel. Therefore, we can conclude that the investigated hydrogel releases a small amount 
of protein during $24 \mathrm{~h}$, under a static experiment and consequently one may indirectly assume that it has high protein adsorption resistance. This aspect is extremely beneficial for further developing hydrogel coatings for, e.g., stent applications with both drug release and protein adsorption resistance, and for ocular applications such as contact or intraocular lenses. However, advanced investigation of different types of proteins, similar to adhesion cell complex and bacterial adhesion and growth is further required to elucidate the eventual protein resistance potential of such hydrogels Future work will also cover investigations in these directions as well as in-depth investigation of the interaction of the hydrogel with other types of proteins.

\subsection{Mineralization studies}

The H9 hydrogel showed very good optical transparency in the visible spectral range (Fig. S8), and because diclofenac is used to treat inflammation of the eye after cataract or corneal refractive surgery, ${ }^{79} \mathrm{H} 9$ could potentially be used for ocular drug delivery applications. According to such potential use, it becomes of uttermost importance to screen the stability of the hydrogel with respect to mineralization phenomena in physiological conditions. Therefore, preliminary mineralization studies of the hydrogels obtained using azelaic acid (H9) have been performed to investigate its behavior in synthetic body fluid (SBF). SEM investigation indicated that the surface morphology typical to amorphous samples did not change after incubation in SBF and no mineral phase was identified on the samples even after 21 days (Fig. 6). Such behavior clearly indicates that the hydrogel does not promote mineralization phenomena. This was also confirmed by EDX analysis, in which no mineral phase (containing calcium of phosphorus) has been detected on the surface of the samples after incubation in SBF. This is an important indication for the potential use of such scaffolds as implantable biomaterials not promoting mineralization/calcification phenomena such is the case of intraocular lenses or devices. 

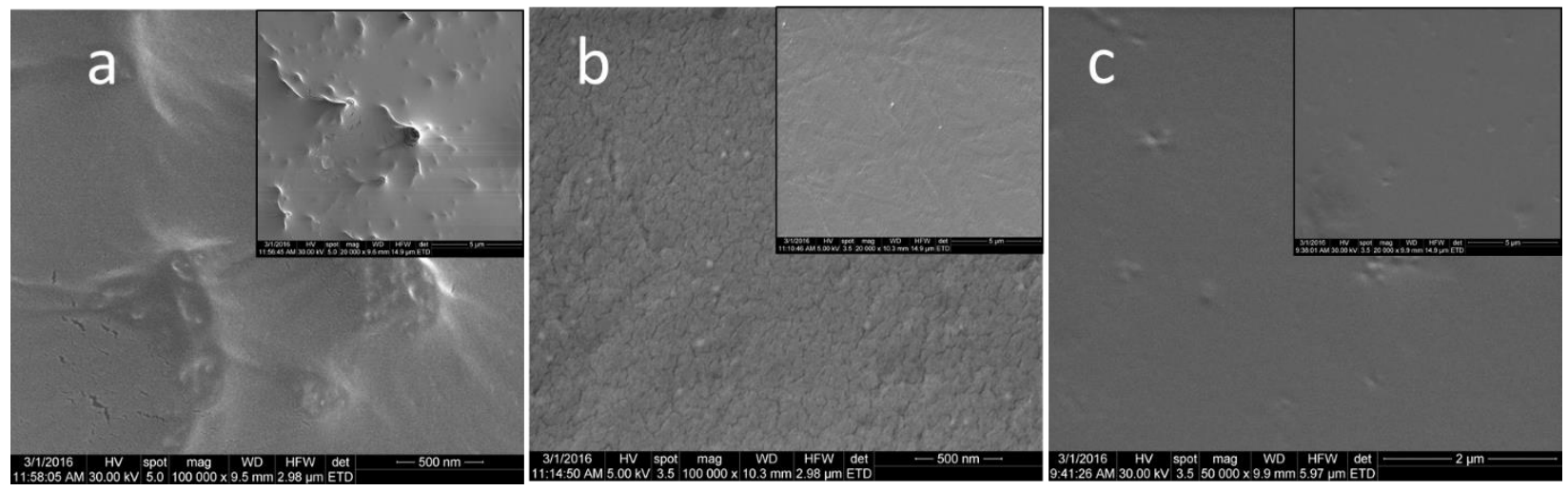

Figure 6. SEM micrographs relevant for the surface of the hydrogel after incubation in SBF: a) after 7 days incubation, b) after 14 days, c) after 21 days. No calcification, no surface fractures nor degradation were noticed after the established time intervals.

\section{CONCLUSIONS}

Novel hydrogels with tunable water uptake were developed using a simple yet robust synthetic strategy by cross-linking a well-defined PiPOx polymer with different dicarboxylic acids. The ESD could be easily tailored by varying the polymer concentration, the length of the cross-linker and/or the COOH/iPOx molar ratio. Swelling studies reveal that the ESD shows an odd-even alternation as a function of the cross-linker length. The polymer gels with odd alkyl ester amide chains have a looser interlayer packing allowing the water molecules to reside between the chains. The hydrogels displayed temperature sensitivity in water and also $\mathrm{pH}$ side-chain degradability, when tested in simulated physiological fluids. Hydrogel H12 crosslinked with dodecanedioic acid displayed a VPTT of $43{ }^{\circ} \mathrm{C}$. The analysis of the swelling process in PBS at pH 7.4 clearly indicated a dependence of diffusion mechanism on the cross-linker length. The swelling exponent was below 0.5 for the hydrogels cross-linked with longer chain dicarboxylic acids, pointing to a Fickian transport mechanism. Moreover, water penetration into the hydrogel followed second-order kinetics, with the swelling rate constant $\mathrm{K}$ decreasing with the increase of the cross-linker length. The rate of drug release at $\mathrm{pH} 7.4$ was affected mainly by drug-hydrogel network interactions since the more hydrophobic DCFNa was released much slower than 5FU. The release rate of both drugs was dependent on the hydrophobicity of the cross-linker. An anomalous (non-Fickian) transport mechanism was evidenced in the case of DCFNa release, while for 5FU the release was controlled only by the diffusion, i.e. Fickian transport mechanism. In vitro cytotoxicity measurements and protein adsorption study demonstrate the non-cytotoxic character of PiPOx hydrogels, and low preliminary adhesion to BSA. The 
mineralization studies showed that PiPOx hydrogel do not promote the mineralization/calcification phenomena.

This work opens new avenues towards developing smart drug delivery systems, make use of the thermosensitivity of the PiPOx hydrogels, and in ophthalmology based on its very good optical transparency and tunable ESD. Future work will also cover investigations on enzymatic degradability of the here reported hydrogels, mechanical testing, as well as the advanced monitoring of their protein interactions.

Supporting information available: The supporting material contains the experimental section, Scheme S1 and the supporting Figures S1-S8.

Acknowledgements: Dr. Jerca acknowledges the Romanian National Authority for Scientific Research (UEFISCDI) for the financial support grant PN-III-P1-1.2-PCCDI no.70/2018 and mobility grants PNIII-P1-1.1-MC-2018-1278 and PN-III-P1-1.1-MC-2018-1263. 


\section{REFERENCES.}

1. Hoffman, A. S. Hydrogels for Biomedical Applications. Adv. Drug Delivery Rev. 2012, 64, 18-23.

2. Ahmed, E. M. Hydrogel: Preparation, Characterization, and Applications: A Review. J. Adv. Res. $\mathbf{2 0 1 5}, 6,105-121$.

3. Caló, E.; Khutoryanskiy, V. V. Biomedical Applications of Hydrogels: A Review of Patents and Commercial Products. Eur. Polym. J. 2015, 65, 252-267.

4. Lin, C.-C.; Metters, A. T. Hydrogels in Controlled Release Formulations: Network Design and Mathematical Modeling. Adv. Drug Delivery Rev. 2006, 58, 1379-1408.

5. Stoddart, A. Hydrogels: A Less Than Swell Time. Nat. Rev. Mater. 2017, 2, 17018.

6. Kopeček, J. Hydrogel Biomaterials: A Smart Future? Biomaterials 2007, 28, 5185-5192.

7. Boateng, J. S.; Matthews, K. H.; Stevens, H. N. E.; Eccleston, G. M. Wound Healing Dressings and Drug Delivery Systems: A Review. J. Pharm. Sci. 2008, 97, 2892-2923.

8. Boateng, J.; Catanzano, O. Advanced Therapeutic Dressings for Effective Wound Healing - A Review. J. Pharm. Sci. 2015, 104, 3653-3680.

9. Zahedi, P.; Rezaeian, I.; Ranaei-Siadat, S.-O.; Jafari, S.-H.; Supaphol, P. A Review on Wound Dressings with an Emphasis on Electrospun Nanofibrous Polymeric Bandages. Polym. Adv. Technol. 2010, 21, 77-95.

10. Lee, K. Y.; Mooney, D. J. Hydrogels for Tissue Engineering. Chem. Rev. 2001, 101, 1869-1880.

11. Drury, J. L.; Mooney, D. J. Hydrogels for Tissue Engineering: Scaffold Design Variables and Applications. Biomaterials 2003, 24, 4337-4351.

12. Jiang, Y.; Chen, J.; Deng, C.; Suuronen, E. J.; Zhong, Z. Click Hydrogels, Microgels and Nanogels: Emerging Platforms for Drug Delivery and Tissue Engineering. Biomaterials 2014, 35, 49694985. 
13. Billiet, T.; Vandenhaute, M.; Schelfhout, J.; Van Vlierberghe, S.; Dubruel, P. A Review of Trends and Limitations in Hydrogel-Rapid Prototyping for Tissue Engineering. Biomaterials 2012, 33, 60206041.

14. Tighe, B. J.; Mann, A. In Biomaterials and Regenerative Medicine in Ophthalmology (Second Edition); Chirila, T. V.; Harkin, D. G., Eds.; Woodhead Publishing: 2016; pp 75-100.

15. Su, X.; Tan, M. J.; Li, Z.; Wong, M.; Rajamani, L.; Lingam, G.; Loh, X. J. Recent Progress in Using Biomaterials as Vitreous Substitutes. Biomacromolecules 2015, 16, 3093-3102.

16. Li, J.; Mooney, D. J. Designing Hydrogels for Controlled Drug Delivery. Nat. Rev. Mater. 2016, $1,16071$.

17. Culver, H. R.; Clegg, J. R.; Peppas, N. A. Analyte-Responsive Hydrogels: Intelligent Materials for Biosensing and Drug Delivery. Acc. Chem. Res. 2017, 50, 170-178.

18. Dargaville, T. R.; Hollier, B. G.; Shokoohmand, A.; Hoogenboom, R. Poly(2-Oxazoline) Hydrogels as Next Generation Three-Dimensional Cell Supports. Cell Adhes. Migr. 2014, 8, 88-93.

19. Lee, D.; Cho, S.; Park, H. S.; Kwon, I. Ocular Drug Delivery through PHEMA-Hydrogel Contact Lenses Co-Loaded with Lipophilic Vitamins. Sci. Rep. 2016, 6, 34194.

20. Lin, C.-C.; Anseth, K. S. PEG Hydrogels for the Controlled Release of Biomolecules in Regenerative Medicine. Pharm. Res. 2009, 26, 631-643.

21. Slaughter, B. V.; Khurshid, S. S.; Fisher, O. Z.; Khademhosseini, A.; Peppas, N. A. Hydrogels in Regenerative Medicine. Adv. Mater. 2009, 21, 3307-3329.

22. Zhu, J.; Marchant, R. E. Design Properties of Hydrogel Tissue-Engineering Scaffolds. Expert Rev. Med. Devices 2011, 8, 607-626.

23. Haq, M. A.; Su, Y.; Wang, D. Mechanical Properties of PNIPAM Based Hydrogels: A Review. Mater. Sci. Eng., C 2017, 70, 842-855. 
24. Parlato, M.; Reichert, S.; Barney, N.; Murphy, W. L. Poly(Ethylene Glycol) Hydrogels with Adaptable Mechanical and Degradation Properties for Use in Biomedical Applications. Macromol. Biosci. 2014, 14, 687-698.

25. Roy, N.; Saha, N. In Hydrogels: Synthesis, Characterization and Applications; Nova Science Publishers: 2012.

26. Teodorescu, M.; Bercea, M. Poly(Vinylpyrrolidone) - A Versatile Polymer for Biomedical and Beyond Medical Applications. Polym.-Plast. Technol. Eng. 2015, 54, 923-943.

27. Awasthi, R.; Manchanda, S.; Das, P.; Velu, V.; Malipeddi, H.; Pabreja, K.; Pinto, T. D. J. A.; Gupta, G.; Dua, K. In Engineering of Biomaterials for Drug Delivery Systems; Parambath, A., Ed.; Woodhead Publishing: 2018; pp 255-272.

28. Ma, S.; Wang, S.; Li, Q.; Leng, Y.; Wang, L.; Hu, G.-H. A Novel Method for Preparing Poly(Vinyl Alcohol) Hydrogels: Preparation, Characterization, and Application. Ind. Eng. Chem. Res. 2017, 56, 79717976.

29. Kamoun, E. A.; Chen, X.; Mohy Eldin, M. S.; Kenawy, E.-R. S. Crosslinked Poly(Vinyl Alcohol) Hydrogels for Wound Dressing Applications: A Review of Remarkably Blended Polymers. Arab. J. Chem. 2015, 8, 1-14.

30. Kumar, A.; Han, S. S. Pva-Based Hydrogels for Tissue Engineering: A Review. Int. J. Polym. Mater. Polym. Biomat. 2017, 66, 159-182.

31. Macková, H.; Plichta, Z.; Hlídková, H.; Sedláček, O.; Konefal, R.; Sadakbayeva, Z.; DuškováSmrčková, M.; Horák, D.; Kubinová, Š. Reductively Degradable Poly(2-Hydroxyethyl Methacrylate) Hydrogels with Oriented Porosity for Tissue Engineering Applications. ACS Appl. Mater. Interfaces 2017, 9, 10544-10553. 
32. Chirila, T. V.; Constable, I. J.; Crawford, G. J.; Vijayasekaran, S.; Thompson, D. E.; Chen, Y.-C.; Fletcher, W. A.; Griffin, B. J. Poly(2-Hydroxyethyl Methacrylate) Sponges as Implant Materials: In Vivo and in Vitro Evaluation of Cellular Invasion. Biomaterials 1993, 14, 26-38.

33. Dalton, P. D.; Flynn, L.; Shoichet, M. S. Manufacture of Poly(2-Hydroxyethyl Methacrylate-CoMethyl Methacrylate) Hydrogel Tubes for Use as Nerve Guidance Channels. Biomaterials 2002, 23, 38433851.

34. Peppas, N. A.; Moynihan, H. J.; Lucht, L. M. The Structure of Highly Crosslinked Poly(2Hydroxyethyl Methacrylate) Hydrogels. J. Biomed. Mater. Res. 1985, 19, 397-411.

35. Hartlieb, M.; Kempe, K.; Schubert, U. S. Covalently Cross-Linked Poly(2-Oxazoline) Materials for Biomedical Applications - from Hydrogels to Self-Assembled and Templated Structures. J. Mat. Chem. B 2015, 3, 526-538.

36. Zahoranová, A.; Kroneková, Z.; Zahoran, M.; Chorvát, D.; Janigová, I.; Kronek, J. Poly(2Oxazoline) Hydrogels Crosslinked with Aliphatic Bis(2-Oxazoline)S: Properties, Cytotoxicity, and Cell Cultivation. J. Polym. Sci., Part A: Polym. Chem. 2016, 54, 1548-1559.

37. Dargaville, T. R.; Park, J.-R.; Hoogenboom, R. Poly(2-Oxazoline) Hydrogels: State-of-the-Art and Emerging Applications. Macromol. Biosci. 2018, 18, 1800070.

38. Shenoi, R. A. In Engineering of Biomaterials for Drug Delivery Systems: Beyond Polyethylene Glycol; Parambath, A., Ed.; Woodhead Publishing: Duxford, United Kingdom, 2018; Chapter 10, pp 273297.

39. Armstrong, J. K. In Pegylated Protein Drugs: Basic Science and Clinical Applications; Veronese, F. M., Ed.; Birkhäuser Basel: Basel, 2009; pp 147-168.

40. Cooperstein, M. A.; Canavan, H. E. Assessment of Cytotoxicity of (N-Isopropyl Acrylamide) and Poly(N-Isopropyl Acrylamide)-Coated Surfaces. Biointerphases 2013, 8, 19. 
41. Adachi, A.; Fukunaga, A.; Hayashi, K.; Kunisada, M.; Horikawa, T. Anaphylaxis to Polyvinylpyrrolidone after Vaginal Application of Povidone-Iodine. Contact Dermatitis 2003, 48, 133136.

42. Alam, A. S.; Parrott, E. L. Effect of Adjuvants on Tackiness of Polyvinylpyrrolidone Film Coating. J. Pharm. Sci. 1972, 61, 265-268.

43. Kato, Y.; Nagao, Y. Effect of Polyvinylpyrrolidone on Sperm Function and Early Embryonic Development Following Intracytoplasmic Sperm Injection in Human Assisted Reproduction. Reprod. Med. Biol. 2012, 11, 165-176.

44. Zhou, W. Y.; Guo, B.; Liu, M.; Liao, R.; Rabie, A. B. M.; Jia, D. Poly(Vinyl Alcohol)/Halloysite Nanotubes Bionanocomposite Films: Properties and in Vitro Osteoblasts and Fibroblasts Response. J. Biomed. Mater. Res., Part A 2010, 93A, 1574-1587.

45. Hassan, C. M.; Peppas, N. A. In Biopolymers · PVA Hydrogels, Anionic Polymerisation Nanocomposites; Springer Berlin Heidelberg: Berlin, Heidelberg, 2000; pp 37-65.

46. Wang, X.; Li, X.; Li, Y.; Zhou, Y.; Fan, C.; Li, W.; Ma, S.; Fan, Y.; Huang, Y.; Li, N.; Liu, Y. Synthesis, Characterization and Biocompatibility of Poly(2-Ethyl-2-Oxazoline)-Poly(D,L-Lactide)Poly(2-Ethyl-2-Oxazoline) Hydrogels. Acta Biomater. 2011, 7, 4149-4159.

47. Jerca, V. V.; Nicolescu, F. A.; Vasilescu, D. S.; Vuluga, D. M. Synthesis of a New Oxazoline Macromonomer for Dispersion Polymerization. Polym. Bull. 2011, 66, 785-796.

48. Verbraeken, B.; Monnery, B. D.; Lava, K.; Hoogenboom, R. The Chemistry of Poly(2Oxazoline)S. Eur. Polym. J. 2017, 88, 451-469.

49. Lorson, T.; Lübtow, M. M.; Wegener, E.; Haider, M. S.; Borova, S.; Nahm, D.; Jordan, R.; Sokolski-Papkov, M.; Kabanov, A. V.; Luxenhofer, R. Poly(2-Oxazoline)S Based Biomaterials: A Comprehensive and Critical Update. Biomaterials 2018, 178, 204-280. 
50. Hartlieb, M.; Pretzel, D.; Englert, C.; Hentschel, M.; Kempe, K.; Gottschaldt, M.; Schubert, U. S. Matrix Supported Poly(2-Oxazoline)-Based Hydrogels for DNA Catch and Release. Biomacromolecules 2014, 15, 1970-1978.

51. Lorson, T.; Jaksch, S.; Lübtow, M. M.; Jüngst, T.; Groll, J.; Lühmann, T.; Luxenhofer, R. A Thermogelling Supramolecular Hydrogel with Sponge-Like Morphology as a Cytocompatible Bioink. Biomacromolecules 2017, 18, 2161-2171.

52. Platen, M.; Mathieu, E.; Lück, S.; Schubel, R.; Jordan, R.; Pautot, S. Poly(2-Oxazoline)-Based Microgel Particles for Neuronal Cell Culture. Biomacromolecules 2015, 16, 1516-1524.

53. Jerca, F. A.; Jerca, V. V.; Anghelache, A. M.; Vuluga, D. M.; Hoogenboom, R. Poly(2Isopropenyl-2-Oxazoline) as a Versatile Platform Towards Thermoresponsive Copolymers. Polym. Chem. 2018, 9, 3473-3478.

54. Feng, H.; Changez, M.; Hong, K.; Mays, J. W.; Kang, N.-G. 2-Isopropenyl-2-Oxazoline: WellDefined Homopolymers and Block Copolymers Via Living Anionic Polymerization. Macromolecules 2017, 50, 54-62.

55. Zhang, N.; Huber, S.; Schulz, A.; Luxenhofer, R.; Jordan, R. Cylindrical Molecular Brushes of Poly(2-Oxazoline)S from 2-Isopropenyl-2-Oxazoline. Macromolecules 2009, 42, 2215-2221.

56. Tomalia, D. A.; Thill, B. P.; Fazio, M. J. Ionic Oligomerization and Polymerization of 2-Alkenyl2-Oxazolines. Polym. J. 1980, 12, 661.

57. Jerca, V. V.; Nicolescu, F. A.; Trusca, R.; Vasile, E.; Baran, A.; Anghel, D. F.; Vasilescu, D. S.; Vuluga, D. M. Oxazoline-Functional Polymer Particles Graft with Azo-Dye. React. Funct. Polym. 2011, $71,373-379$. 
58. Spiridon, M. C.; Jerca, F. A.; Jerca, V. V.; Vasilescu, D. S.; Vuluga, D. M. 2-Oxazoline Based Photo-Responsive Azo-Polymers. Synthesis, Characterization and Isomerization Kinetics. Eur. Polym. J. 2013, 49, 452-463.

59. Weber, C.; Neuwirth, T.; Kempe, K.; Ozkahraman, B.; Tamahkar, E.; Mert, H.; Becer, C. R.; Schubert, U. S. 2-Isopropenyl-2-Oxazoline: A Versatile Monomer for Functionalization of Polymers Obtained Via Raft. Macromolecules 2012, 45, 20-27.

60. Zhang, N.; Salzinger, S.; Soller, B. S.; Rieger, B. Rare Earth Metal-Mediated Group-Transfer Polymerization: From Defined Polymer Microstructures to High-Precision Nano-Scaled Objects. J. Am. Chem. Soc. 2013, 135, 8810-8813.

61. Altenbuchner, P. T.; Soller, B. S.; Kissling, S.; Bachmann, T.; Kronast, A.; Vagin, S. I.; Rieger, B. Versatile 2-Methoxyethylaminobis(Phenolate)Yttrium Catalysts: Catalytic Precision Polymerization of Polar Monomers Via Rare Earth Metal-Mediated Group Transfer Polymerization. Macromolecules 2014, 47, $7742-7749$.

62. Kroneková, Z.; Mikulec, M.; Petrenčíková, N.; Paulovičová, E.; Paulovičová, L.; Jančinová, V.; Nosál, R.; Reddy, P. S.; Shimoga, G. D.; Chorvát, D.; Kronek, J. Ex Vivo and in Vitro Studies on the Cytotoxicity and Immunomodulative Properties of Poly(2-Isopropenyl-2-Oxazoline) as a New Type of Biomedical Polymer. Macromol. Biosci. 2016, 16, 1200-1211.

63. Jerca, F. A.; Jerca, V. V.; Hoogenboom, R. Well-Defined Thermoresponsive Polymethacrylamide Copolymers with Ester Pendent Groups through One-Pot Statistical Postpolymerization Modification of Poly(2-Isopropenyl-2-Oxazoline) with Multiple Carboxylic Acids. J. Polym. Sci., Part A: Polym. Chem. 2018, DOI:10.1002/pola.29188.

64. Azevedo, H. S.; Reis, R. L. In Biodegradable Systems in Tissue Engineering and Regenerative Medicine; 2004; pp 177-201. 
65. Teodorescu, M.; Cursaru, B.; Stanescu, P. O. Swelling and Diffusion Characteristics of Hydrogels Synthesized from Diepoxy-Terminated Poly(Ethylene Glycol)S and Aliphatic Polyamines. Soft Mater. 2010, 8, 288-306.

66. Fu, Y.; Kao, W. J. Drug Release Kinetics and Transport Mechanisms of Non-Degradable and Degradable Polymeric Delivery Systems. Expert Opin. Drug Deliv. 2010, 7, 429-444.

67. Wang, R.; Johnson, J. A.; Olsen, B. D. Odd-Even Effect of Junction Functionality on the Topology and Elasticity of Polymer Networks. Macromolecules 2017, 50, 2556-2564.

68. Zhang, Q.; Zha, L.; Ma, J.; Liang, B. A Novel Route to Prepare pH- and Temperature-Sensitive Nanogels Via a Semibatch Process. J. Colloid Interface Sci. 2009, 330, 330-336.

69. Liu, Q.; Zhang, P.; Qing, A.; Lan, Y.; Lu, M. Poly(N-Isopropylacrylamide) Hydrogels with Improved Shrinking Kinetics by Raft Polymerization. Polymer 2006, 47, 2330-2336.

70. Imaz, A.; Forcada, J. N-Vinylcaprolactam-Based Microgels: Synthesis and Characterization. $J$. Polym. Sci., Part A: Polym. Chem. 2008, 46, 2510-2524.

71. Rihova, B.; Bilej, M.; Vetvicka, V.; Ulbrich, K.; Strohalm, J.; Kopecek, J.; Duncan, R. Biocompatibility of N-(2-Hydroxypropyl) Methacrylamide Copolymers Containing Adriamycin: Immunogenicity, and Effect on Haematopoietic Stem Cells in Bone Marrow in Vivo and Mouse Splenocytes and Human Peripheral Blood Lymphocytes in Vitro. Biomaterials 1989, 10, 335-342.

72. Seymour, L. W.; Duncan, R.; Strohalm, J.; Kopeček, J. Effect of Molecular Weight (Mw) of N(2-Hydroxypropyl)Methacrylamide Copolymers on Body Distribution and Rate of Excretion after Subcutaneous, Intraperitoneal, and Intravenous Administration to Rats. J. Biomed. Mater. Res. 1987, 21, $1341-1358$.

73. Mandal, B. B.; Kapoor, S.; Kundu, S. C. Silk Fibroin/Polyacrylamide Semi-Interpenetrating Network Hydrogels for Controlled Drug Release. Biomaterials 2009, 30, 2826-2836. 
74. Brazel, C. S.; Peppas, N. A. Mechanisms of Solute and Drug Transport in Relaxing, Swellable, Hydrophilic Glassy Polymers. Polymer 1999, 40, 3383-3398.

75. Schott, H. Swelling Kinetics of Polymers. J. Macromol. Sci., Part B: Phys. 1992, 31, 1-9.

76. Jerca, V. V.; Jerca, F. A.; Rau, I.; Manea, A. M.; Vuluga, D. M.; Kajzar, F. Advances in Understanding the Photoresponsive Behavior of Azobenzenes Substituted with Strong Electron Withdrawing Groups. Opt. Mater. 2015, 48, 160-164.

77. Ritger, P. L.; Peppas, N. A. A Simple Equation for Description of Solute Release II. Fickian and Anomalous Release from Swellable Devices. J. Control. Release 1987, 5, 37-42.

78. Baker, S. J. In Comprehensive Medicinal Chemistry II; Taylor, J. B.; Triggle, D. J., Eds.; Elsevier: Oxford, 2007; pp 957-968.

79. Goa, K. L.; Chrisp, P. Ocular Diclofenac. Drugs Aging 1992, 2, 473-486. 
TOC GRAPHIC:

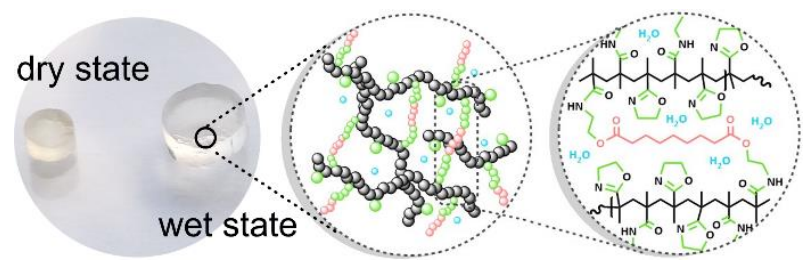

